voluntarily, and by the formation of corporations or holding companies. This process has evoked a mixed reception, and a parer by Mr. G. M. Gill records how the holding companies have already given technical and financial assistance to many small isolated companies, enabling them to pursue a more progressive policy.

\section{A Meteorological Chronology}

Geophysical. Memoir No. 70 of the Meteorological Office, by C. E. Britton, is entitled "A Meteorological Chronology to A.D. 1450". It is a symposium of various compilations of historical references to the weather of the British Isles, and a full bibliography of these is given at the end of the memoir. The main body of the work is in diary form, each year for which any notable meteorological event has been recorded appearing in turn, with particulars of such events. The first year is 2668 B.c., but it is pointed out that these very early dates, derived from such sources as the Irish Chronicles, are "largely legendary", being probably in the nature of oral traditions. The events chronicled in these years are chiefly the overflowing of various Irish lakes and rivers. The traditional period is followed by the Roman period, and the author points out that the very large number of meteorological events assigned to those times by various annalists and chroniclers do not appear to have been recorded by any contemporary writer; many indeed aprear for the first time on record in the eighteenth century, with no information from the chronicler as to where the information was obtained. A typical specimen of this class of record is that of Dr. Thomas Short of Sheffield, who published a "General Chronological History of the Air, Weather, Seasons, Meteors, Etc." in two volumes in 1749-a work that occupied the worthy doctor for fifteen years. Without disputing the interest attaching to a work of the character of this symposium, one may doubt if its scientific value for meteorology is appreciable. It is amusing to notice the sobering of the record in its later years-the gradual dying out of such events as several days' downpour of blood, for example-with its suggestion that a very large part of the events before, say, A.D. 1000, are pure inventions or unrecognizable distortions of happenings not so very different from those of modern times.

\section{A Theory of Atomic Structure}

OUR attention has been directed to a theory of atomic structure which has been elaborated by Mr. C. F. Krafft, of Washington, D.C., and published by him in book form under the title "The Mechanistic Autonomy of Nature, with 1935 and 1936 Supplements". In this theory the atomic nucleus has been discarded in favour of a system of vortex rings arranged in the form of polygons, which may break up into chemically active groups and then fuse with other similar groups to form larger polygons. Thus the valency 'bonds' are made up of protons as well as electrons and the structural centre of the atom is apparently void. Whether this theory will survive the critical examination of the physicist is not certain, but the author claims that it enables him to interpret the chemical structures of compounds like diborane $\mathrm{B}_{2} \mathrm{H}_{6}$, etc., which are apparently anomalous. $\mathrm{He}$ asserts that "the very existence of diborane flatly disproves the entire nuclear theory of atomic structure, and not even its endorsement by high authorities can save it". In view of this bold statement, it is interesting to recall the fact that this very problem was brilliantly solved quite recently by Dr. E. Wiberg, an account of whose work on the subject was published in NATURE of February 27, p. 381.

\section{Storage Battery Cars}

RECENT progress in the design of storage batteries has turned the attention of traction engineers to developing storage battery cars for use on rails. These cars are very trustworthy in service and simple to operate. The maintenance costs of the equipment are much lower than when Diesel engines are used. Battery maintenance is usually carried out by the manufacturers on the basis of the number of miles run, and the cost depends on the current price of lead. The 'standstill' charges affect about ten per cent of the total stock in service, and due to battery overhauls they are usually out of commission for three or four days during the year. According to Electrical Industries of May 19, charging the batteries is very simple. It is carried out at night by connecting them to a power supply, and when fully charged the current is automatically switched off. At the terminal points of the system the whole battery can be replaced in a quarter of an hour. The distance that can be covered in a single battery charge is now 185 miles. Trials on the German State Railways show that some cars can travel for 250 miles non-stop at 25 m.p.h., and half that distance can be covered at 40 m.p.h. In the first case the power consumption is 18 watt hours per ton mile and in the second 26 watt hours per ton mile. At the present time, there are 170 cars on the German State Railways and their average speed is between 37 and 47 m.p.h. The average yearly car mileage during 1935 was 38,500 . In Poland, France and the north of Italy, storage battery cars, mostly of the double bogie type, are run on tramways and suburban lines. Each car carries about a hundred passengers and has four motors giving a total of about 180 horse-power.

\section{The National Physical Laboratory.}

THE annual report of the Laboratory for 1936 is presented in a new form. Hitherto it has been a quarto volume of about 250 pages, well illustrated and cost $12 s$.; now it is royal octavo of 144 pages without illustrations but costs only $2 s .6 d$. The saving of space has been secured by the omission of detail of limited interest. The death of Sir Joseph Petavel, the director, was referred to in the last report and until the appointment of a successor Sir Frank Smith was in charge. Several members of the staff have been transferred to other Government Departments or have been absorbed by industry. Many of the senior members have taken part in conferences at home and abroad and have lectured in provincial centres of industry on the work of the 